Revista Venezolana de Gerencia (RVG)

Año 13. No 44, 2008, 543 - 555

Universidad del Zulia (LUZ) • ISSN 1315-9984

\title{
Empresas integradoras de México: Influencias europeas*
}

\author{
Rodríguez Monroy, Carlos** \\ Fernández Chalé, Lizbeth Noemí ${ }^{* * *}$
}

\begin{abstract}
Resumen
La apertura comercial de México que se da en los 90's, hizo que el país abandonara un sistema comercial endógeno fuertemente controlado y protegido, tomando como ejemplo la experiencia de otros países. El gobierno promovió nuevas formas de asociación productiva, entre ellas, la "Empresa Integradora". El trabajo plantea un análisis de la figura "Empresas Integradoras", a través del derecho comparado, examinando figuras asociativas europeas similares. La investigación fundamenta su análisis en la revisión y estudio de la literatura referida al tema, obteniendo una visión más amplia del marco jurídico en el que se desarrolla la figura asociativa de la Empresa Integradora, en México. Los resultados indican que la Empresa Integradora mexicana es una figura inspirada en el asociacionismo europeo, pero desafortunadamente carece de un programa fiscal propio a diferencia de sus homólogas europeas, además de presentar inconsistencias en sus conceptos, generando confusión en su comprensión. Visto lo anterior, es así como podemos conocer otras realidades similares a la mexicana, estableciendo ciertos paralelismos jurídicos que ayuden a entender y mejorar la producción propia.
\end{abstract}

Palabras clave: Empresas integradoras, agrupaciones de interés económico, distrito industrial, consorcio, pequeñas y medianas empresas (pymes)

Recibido: 28-03-08. Aceptado: 20-11-08

* Este trabajo constituye un producto de la tesis doctoral "Asociación Empresarial y las PYME's en México. La participación frente a estos agrupamientos. Definición de un Modelo", de la Universidad Politécnica de Madrid.

** Doctor Ingeniero Industrial, Profesor Titular investigador de la Universidad Politécnica de Madrid. Licenciado en Ciencias Económicas y Empresariales, Licenciado en Derecho, Licenciado en Sociología. Coordinador de programas de Maestría y de Doctorado.

E-mail: crmonroy@ingor.etsii.upm.es

*** Ingeniero industrial, Maestría en Planificación Industrial, cursa estudios de Doctorado en Administración de Empresas. Profesor investigador de la Universidad Autónoma de Yucatán.

E-mail: fchale@uady.mx 
Empresas integradoras de México: Influencias europeas

Rodríguez Monroy, Carlos y Fernández Chalé, Lizbeth Noemí

\section{The Integrative Companies of Mexico: European Influence Abstract}

The commercial opening of Mexico that took place in the 90's, induced the country to abandon a strongly controlled and protected endogenous commercial system, using the experience of other countries. As an example, the government promoted new forms of productive affiliation and among them the "integrative companies". This research work derives from an analysis of the figure of the integrative companies, through the study of compared legislation, examining similar European associative models. The investigation bases the analysis on the literature review of the referred topic, obtaining a wider vision of the legislative framework than the one that develops the associative figure of integrative companies in Mexico. The results indicate that the Mexican integrative company is a figure that has been inspired by the European "asociacionismo" (associativeness), but unfortunately it lacks a fiscal program of its own in contrast to the homologous European models. In addition, it presents conceptual inconsistencies generating confusion in its comprehension. In view of these considerations, this is how we can know other realities similar to the Mexican, establishing certain legislative parallelisms that help us to understand and to improve the country's production.

Key words: Integrative Companies, Groups of Economic Interest, Industrial Districts, Consortia, Micro, Small and Medium Enterprises (MSME's).

\section{Introducción}

La apertura comercial desarrollada en México en los años noventa ${ }^{1}$, iniciada con la firma del Tratado de Libre Comercio con los Estados Unidos de América y Canadá (TLCAN), supuso un cambio radical en el país: México abandonaba un sistema productivo sustentado en los monopolios estatales y paraestatales, un sistema comercial endógeno fuertemente controlado y protegido, y un sistema social basado en una revolución garante del subsidio compensatorio.

En este contexto socioeconómico, la firma del TLCAN establece las líneas maestras de la nueva política económica en torno a la legislación sobre la Inversión Extrajera Directa (IED) en México. El gobierno fue eliminando barrera tras barrera, a la IED (Secretaría de Comercio y Fomento Industrial, SECOFI, 1993b, 1996), hasta permitir a los inversionistas extranjeros adquirir el control del $100 \%$ del capital social en bancos nacionales (Secretaría de Hacienda y Crédito Público, 1999).

Paralelamente, se promocionaron formas de asociación productiva, tomando como ejemplo la experiencia de otros países con economía abiertas. En este entorno, surge el decreto presidencial del

1 Tras la firma del TLC de América del Norte el 17 de diciembre de 1992 (y que entró en vigor el $1^{\circ}$ de enero de 1994), México suscribió tratados de libre comercio con los siguientes países: Con Costa Rica, Bolivia, Colombia y Venezuela ( $1^{\circ}$ de enero de 1995$)$; con Nicaragua ( $1^{\circ}$ de julio de 1998$)$; con Chile $\left(1^{\circ} \mathrm{de}\right.$ agosto de 1999); con la Unión Europea e Israel ( ${ }^{\circ}$ de julio de 2000); con el Salvador, Guatemala ( 15 de marzo de 2001) y Honduras ( $1^{\circ}$ de junio de 2001); con Islandia, Noruega, Suiza, y Liechtenstein $\left(1^{\circ} \mathrm{de}\right.$ julio de 2001); con Uruguay (15 de julio de 2004); y con Japón (1 de julio de 2005). 
siete de mayo de 1993, que promueve la creación de Empresas Integradoras (EI) (Secretaría de Hacienda y Crédito Público, 1993a). Dicha figura societaria (que no está tipificada ni en el Código de Comercio ni en la Ley General de Sociedades Mercantiles), tiene por objeto la integración o agrupación de varias empresas para crear una nueva, y que ésta "realice gestiones y promociones orientadas a modernizar y ampliar la participación de las empresas agrupadas/integradas en todos los ámbitos de la vida nacional".

Este interés por asociar empresas, especialmente micros, pequeñas y medianas, no es nuevo en México. Como antecedente inmediato, tenemos que en 1988, la ley Federal para el Fomento de la Microindustria, planteaba la asociación, cuando menos para "obtener financiamientos, establecer sistemas de ventas y compras en común de materias primas y productos $y$, en su caso, prestación de servicios de subcontratación y maquila" (SECOFI, 1988).

En relación al decreto presidencial de mayo de 1993, que promueve la creación de empresas integradoras (EI), algunos analistas opinan que dicha figura asociativa, está inspirada en el fenómeno socio-productivo de los distritos industriales italianos, el cual a su vez gira en torno a la figura contractual del consorcio como base de asociación empresarial. En este artículo se aportan datos en el sentido que las "empresas integradoras mexicanas" tienen una identidad propia, bastante diferente de la figura italiana, pero guarda no pocas semejanzas con la figura de la Unión Europea, conocida como agrupación de interés económico.

\section{Del Consorcio Italiano a la Empresa Integradora Mexicana}

Autores nacionales que han realizado estudios sobre el tema (Orozco, 1999; Perera, 2000; Hualde, 2002), consideran que el fenómeno de los distritos industriales italianos, y en particular, la figura asociativa conocida como el consorcio italiano, debe ser considerado como el antecedente socioeconómico, en el que los legisladores mexicanos se inspiraron para redactar el decreto 07/05/1993, por el que se promueve en México la creación de empresas integradoras.

En el mismo sentido, comparte opinión Rueda Peiró (1997), quien afirma que dicho decreto, "está basado en una figura asociativa que ha tenido un desarrollo exitoso en Italia" (Rueda Peiró, 1997: 10); y añade que "...si bien es fácil importar el esquema de empresas integradoras basado en la figura italiana de los consorcios, no es fácil importar o crear de la noche a la mañana todas las condiciones que llevaron a la transformación de una situación muy difícil en otra de prosperidad..." (Rueda, 1997:38).

Abundando sobre el tema y siempre dentro de la misma corriente teórica, Perera Sabido (2000:24) por su parte, afirma que el modelo de empresa integradora, como se pretende manejar en México actualmente, surgió de un modelo adoptado en la economía italiana, modelo que explica como el conjunto de las micro, pequeñas y medianas empresas que se integran para fortalecer el aparato productivo incrementando la capacidad de producción y negociación en los mercados.

El texto del decreto presidencial promulgado en México, propone la mo- 
Empresas integradoras de México: Influencias europeas

Rodríguez Monroy, Carlos y Fernández Chalé, Lizbeth Noemí

dernización de las micro, pequeñas y medianas empresas (pymes), con "especial atención a los programas de organización interempresarial que faciliten el trabajo en equipo e incrementen su capacidad de negociación en los mercados", y promueve "la organización interempresarial para la formación de empresas integradoras en industrias micro, pequeñas y medianas en ramas y regiones con potencial exportador".

En el caso italiano, las organizaciones interempresariales agrupadas en ramas y regiones, conformando redes, es una constante. Durante más de cincuenta años este modelo ha sido clave en el éxito de las pymes y cooperativas italianas: las empresas, en especial pymes, se han ido integrando entre sí a través de consorcios; estos a su vez, han ido conformando la base productiva de ciertas zonas geográficas socioeconómicas, que al unirse entre sí, a su vez han constituido, los distritos industriales (regiones industriales).

Si bien existen semejanzas en los objetivos de ambas figuras jurídicas, (por ejemplo, potenciar pymes mediante figuras asociativas; crear redes industriales; establecer regiones industriales) las herramientas para alcanzarlos han sido disparejas: el consorcio italiano presenta más diferencias que semejanzas, con las empresas integradoras mexicanas. Y diferencias tan claras, como el hecho que existiendo en México la figura del consorcio, los legisladores no optaron por ésta, para promover, constituir y regular la asociación productiva de PYMES.

Una primera diferencia es la obligación impuesta a los consorciados (los asociados por el contrato del consorcio), "a permitir los controles y las inspeccio- nes por parte de los órganos previstos en el contrato, con el fin de verificar el exacto cumplimiento de las obligaciones asumidas" (II Codice Civile Italiano, 1942).

Esto es: los consorciados pueden ser inspeccionados por el consorcio, que no sólo supervisa sus actividades, sino que además puede obligarles al cumplimiento de las condiciones del contrato consorcial y de aquellas disposiciones aprobadas por el consorcio en atribución de sus funciones; las empresas asociadas en este esquema ven limitada su autonomía, "por la disciplina que el consorcio implica en la actividad de sus miembros, garantizada a través de un control efectivo $y$ de una inspección que sobre las empresas miembros se ejerce" (Monge, 1993).

Esta facultad directiva no está contemplada por el legislador en la E.I. mexicana; de hecho no existe ninguna estructura orgánica en la E.I. que ejerza un poder jerárquico de similares características al consorcio italiano.

En el caso mexicano, la E.I. tiene asignada una clara y específica función: actuar como institución auxiliar apoyando a los socios, coordinando voluntades, iniciativas, esfuerzos y proyectos, pero de ningún modo, con poder para controlar las actividades productivas de los socios/integrados, menos aún con la capacidad necesaria para inspeccionar dichas actividades, y por supuesto, carentes totalmente de potestad sancionadora.

Obviamente, los socios/integrados pueden pactar en el documento constitutivo de la E.I., ciertas reglas que permitan o faculten una potestad jerárquica a un órgano específico para actuar en consecuencia, subordinando así los intereses particulares de los socios al colectivo de 
la E.I., aunque como se observa en el decreto, éste no parece ser el objetivo del legislador, quien expresa claramente que deben ser prestadoras de servicios especializados de apoyo (art. $4^{\circ}$. 1 del decreto 07/05/93).

Otra de las diferencias que marcan la identidad de la E.I. con respecto al consorcio, es precisamente su naturaleza asociativa: la El es una sociedad mercantil, mientras que el consorcio es civil. Lo anterior se confirma con la legislación propia que dispone la E.I., (SECOFI, 1993a), y como supletoria, la Ley General de Sociedades Mercantiles y el Código de Comercio.

El consorcio en Italia, es una figura contractual, un contrato, regulado por el Código Civil; dicho código señala como contrato de consorcio cuando, "varios empresarios instituyen una organización común para la disciplina o para el desarrollo de determinadas fases de sus respectivas empresas" (II Codice Civile Italiano, 1942).

Como se aprecia, el consorcio empresarial en Italia es una figura asociativa y está considerado como empresa mutualista pura; por el contrario, las E.I. en México, son consideradas sociedades privadas, de carácter mercantiles, y en la que sus socios responden según su participación accionarial.
Si bien el texto regulador de las E.I., no establece un concepto de esta figura asociativa productiva, podemos inferir algunas ideas a través de los requisitos que debe cumplir así como las funciones que debe realizar. Teniendo en cuenta lo establecido en el mencionado decreto, la E.I. es "aquella persona moral cuyo objeto social preponderante será la prestación de servicios especializados a cuatro micro, pequeñas o medianas empresas como mínimo, de quienes integrará su capital social por un valor no inferior a 50,000.00 pesos y de quienes obtendrá como mínimo el 90\% de sus ingresos".

Se tiene por tanto, como ideas centrales:

- Es una sociedad mercantil, con personalidad propia.

- Carácter auxiliar, no competitivo ni sustituto de la actividad principal de sus socios.

- Conformada por sociedades mercantiles y/o instituciones civiles (el 75\% del capital social deberá ser aportado por pymes).

- Sin ánimo de lucro; pero puede obtener de terceros, hasta el $10 \%$ de sus ingresos ${ }^{2}$.

2 En el Decreto Presidencial del 07/05/1993, el artículo $4^{\circ}$ establecía los requisitos que debían cumplir las empresas para obtener el registro como E.I., y el párrafo $V$ de este artículo indicaba que este tipo de empresas los únicos ingresos que podía percibir, eran por "por concepto de cuotas, comisiones y prestación de servicios" siendo las socias/ integradas, quienes debían satisfacer estos gastos, por, "ser usuarias de los servicios que presta la empresa integradora", según establece el párrafo II de este mismo artículo.

Tras la reforma del 30 de mayo de 1995 , se modifica el párrafo $V$ del artículo $4^{\circ}$, permitiéndole a la empresa integradora "obtener ingresos por otros conceptos, siempre que éstos representen como máximo un 10 por ciento de sus ingresos totales". 
Empresas integradoras de México: Influencias europeas

Rodríguez Monroy, Carlos y Fernández Chalé, Lizbeth Noemí

\section{La empresa integradora y la agrupación de interés económico}

En la Unión Europea existe la figura societaria conocida como agrupación de interés económico. Cada país miembro regula dicha figura jurídica incorporándola a su legislación tomando como base la normativa europea. Para el caso que nos ocupa, utilizaremos el modelo español, el cual está regulado por la Ley de Agrupaciones de Interés Económico, A.I.E. (Jefatura del Estado, 1991). De la lectura de la Ley A.I.E., se observan las siguientes ideas centrales:

- Es una sociedad mercantil, con personalidad propia.

- Carácter auxiliar, no competitivo ni sustitutivo de la actividad principal de sus socios ${ }^{3}$.

- Conformada por sociedades mercantiles y/o instituciones civiles.

- Sin ánimo de lucro para sí misma ${ }^{4}$.

Esta ley regula la creación de una nueva sociedad mercantil integrada por otras sociedades (mercantiles preferen- temente), que tiene como finalidad "facilitar el desarrollo o mejora de los resultados de la actividad de sus socios", y como objetivo "realizar una actividad económica auxiliar de la que desarrollan sus socios" (Jefatura del Estado, 1991).

El antecedente de esta ley, es el Reglamento de la Agrupación Europea de Interés Económico (AEIE), cuya objetivo es favorecer "una cooperación entre pequeñas y medianas empresas, en la medida en que las coloca en situación de trabajar de manera más racional y aumentar su productividad y competitividad en un mercado más amplio" (Gómez, 1993). Dicho reglamento nace, para facilitar la cooperación y asociación de empresas de distintos países miembros de la Unión Europea, para hacer frente a los grandes corporativos internacionales; por ende, la legislación nacional española, en su versión legislativa de la A.I.E., también incorpora la posibilidad que empresas de otros países puedan ser socios de una A.I.E. española. ${ }^{5}$

Esta agrupación empresarial es horizontal, "sin que esta conlleve pérdida

3 El objeto de las empresas de Agrupación de Interés económico queda establecido en el artículo $3^{\circ}$ de la Ley 12/1991, cuando señala que se "limitará exclusivamente a una actividad económica auxiliar de la que desarrollen sus socios". Asimismo la ley le prohíbe explícitamente "poseer directa o indirectamente participaciones en sociedades que sean miembros suyos", también le prohíbe dirigir o controlar directa o indirectamente las actividades de sus socios o de terceros".

4 El artículo $2^{\circ}$ de la Ley 12/1991 de Agrupaciones de Interés Económico, establece la finalidad de éstas, la cual es facilitar el desarrollo o mejorar los resultados de la actividad de sus socios, y en el párrafo Il de este mismo artículo subraya que este tipo de empresas no tienen ánimo de lucro para si misma.

5 El artículo $28^{\circ}$ de la Ley $12 / 1991$ de 29 de abril admite la posibilidad que empresas europeas sean socias de una A.I.E. española, al establecer como condición administrativa "que los socios residentes en España de Agrupaciones de interés económico llevarán en sus registros contables cuentas perfectamente diferenciadas....". 
de autonomía jurídica o económica”, e impide a los socios "poder dominar al resto de sus asociadas" (Monge, 1993). Aquí hay una primera coincidencia con las empresas integradoras de México: tanto las El como las AIE, no pueden participar ni directa ni indirectamente de las empresas que las agrupan y/o integran (SECOFI, 1993a; Jefatura del Estado 1991) (Ver Cuadro 1).

Otra coincidencia es la ausencia del ánimo de lucro. La AIE subraya que la nueva empresa, no puede competir en las actividades de sus socios, ni sustituirlas, debido a la prohibición expresa del ánimo de lucro para si misma (Jefatura del Estado, 1991).

En el caso de la E.I., inicialmente (decreto 07-05-1993) se regía por la ausencia de ánimo de lucro; con la reforma del 30 de mayo de 1995, puede obtener ingresos de terceros, hasta por el $10 \%$ de sus ingresos totales; este ingreso adicional ratifica el objetivo prioritario, que es prestar servicios especializados a sus socios/integrados y de quienes obtiene como mínimo el $90 \%$ de sus ingresos.

El decreto reformador de 1995 justifica esa ampliación de actividades frente a terceros, en la obtención del máximo rendimiento de la capacidad productiva de la E.I., la promoción de servicios para captar a nuevos socios/integrados, y por supuesto comercializar una parte de sus servicios a terceros. Este decreto también facilita el acceso de instituciones de la banca de desarrollo, y de cualquier otro socio, p. ej., empresas "no pymes", al capital social de la E.I.; en ningún caso, la participación rebasará el $25 \%$ del capital social.
Específicamente dice el decreto, que: "Podrán participar en el capital de las empresas integradoras las instituciones de la banca de desarrollo, el Fondo Nacional de Empresas de Solidaridad, y en general, cualquier otro socio, siempre y cuando la participación de las empresas integradas represente por lo menos un $75 \%$ del capital social de la integradora" (SECOFI, 1995).

Con la expresión, "cualquier otro socio", las El están abiertas a cualquier empresa no pyme; este nuevo tipo de socio puede participar hasta con el $25 \%$ del capital social (las pymes pueden tener hasta el 30\%).

Esta reforma elimina dos candados que marcaban las E.I., a saber

1. (1993) No pueden tener ánimo de lucro; (1995) pueden tener animo de lucro para si mismas, como máximo en un $10 \%$ del total de sus ingresos.

2. (1993) Sólo pymes pueden ser socios; (1995) El Estado y empresas no pymes, pueden ser socios hasta en el $25 \%$ de su capital social.

Las E.I. y las AIE, tienen la misma finalidad. En las AIE, se trata de "facilitar el desarrollo o mejora de los resultados de la actividad de sus socios", y su objeto es realizar exclusivamente "una actividad económica auxiliar de la que desarrollen sus socios" (España. Jefatura del Estado, 1991); el objeto de las E.I. es "la prestación de servicios especializados de apoyo a las empresas micro, pequeñas y medianas integradas, esto es, a sus socios; servicios que tienen carácter auxiliar en relación a la actividad principal de los socios (SECOFI, 1993a).

La Ley 12/1991 no recoge una lista de servicios auxiliares (carácter técnico, 
Empresas integradoras de México: Influencias europeas

Rodríguez Monroy, Carlos y Fernández Chalé, Lizbeth Noemí

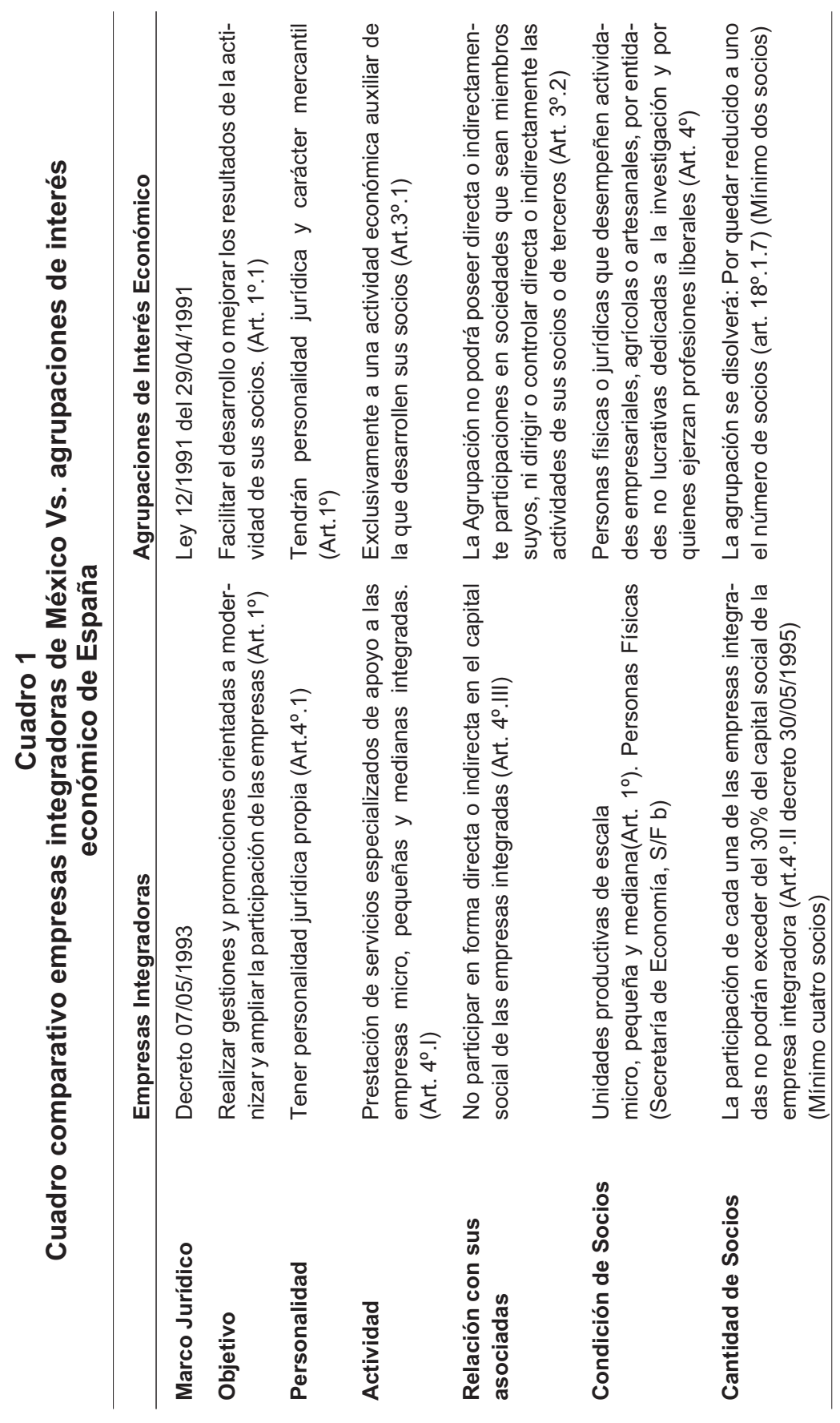




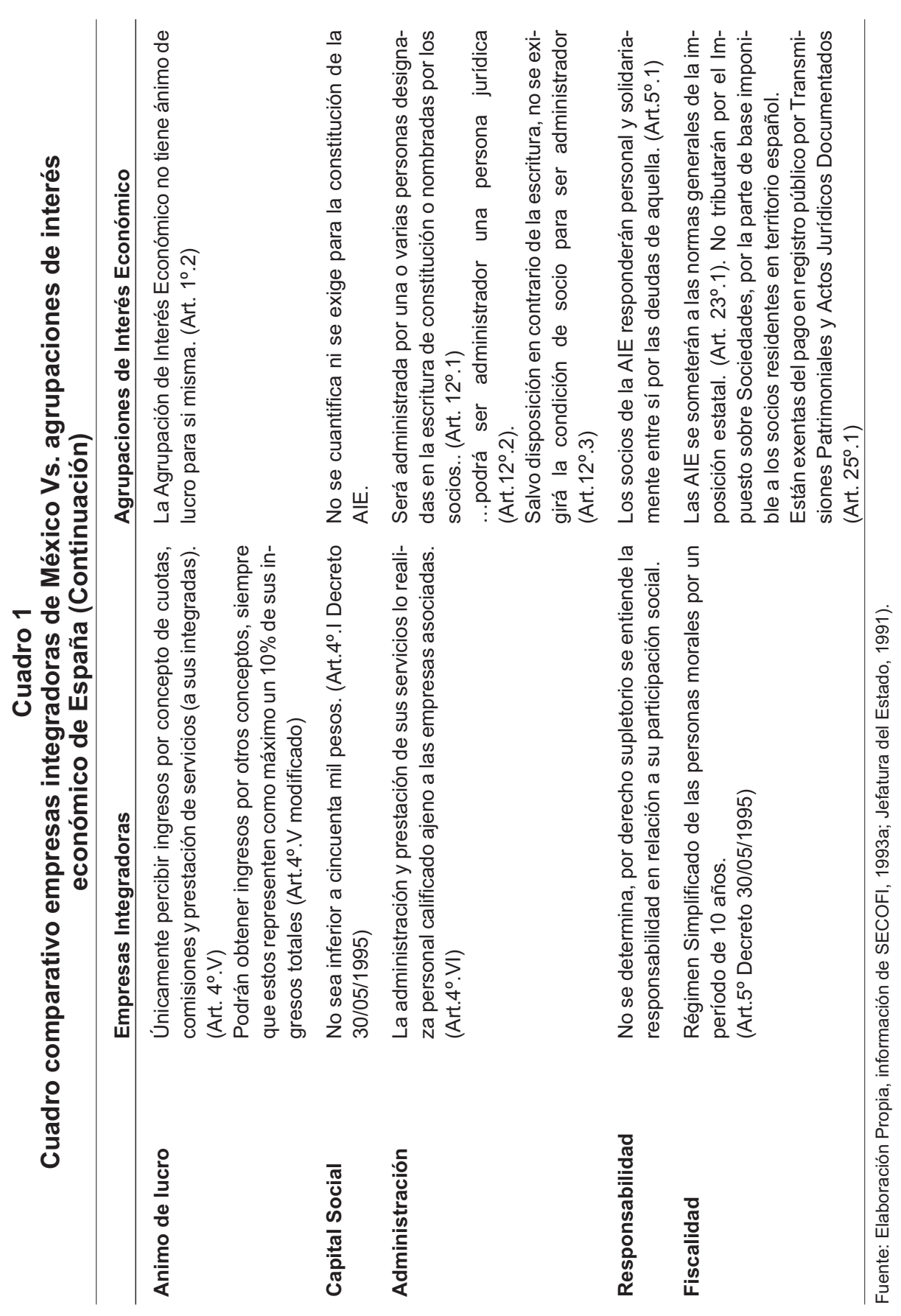


Empresas integradoras de México: Influencias europeas

Rodríguez Monroy, Carlos y Fernández Chalé, Lizbeth Noemí

comercial, industrial, administrativo), pero podrían señalarse como ejemplos:

- Especialización de las empresas miembros.

- Servicio de Estudio común.

- Marcas y etiquetas comunes.

- Proyectos de I+D.

- Prospección de mercados.

- Combinación de actividades para presentar ofertas de gran consideración.

- Participación y solicitud conjunta en programas y subvenciones de la UE ${ }^{6}$.

Por su parte el Decreto 07/05/1993

y su reforma de 30/05/1995, enumeran una serie de servicios especializados, de carácter auxiliar. Dichos servicios son los siguientes:

1. Servicios tecnológicos.

2. Servicios de promoción y comercialización, de los productos y servicios de los socios (este último párrafo fue añadido en la reforma del 30/05/ 1995).

3. Servicios de diseño, identificadores de productos, bienes y servicios.

4. Servicios de subcontratación de productos y procesos industriales, para complementar cadenas productivas.

5. Servicios de gestión de financiamiento en condiciones de fomento.

6. Servicios de gestión de compra en común, de materias primas, tecnología e insumos varios.

7. Servicios de aprovechamiento de residuos industriales, especialmente reciclaje (este punto fue añadido en la reforma del 30/05/1995).
8. Otros servicios que se requieran para el óptimo desempeño de las empresas integradas.

En el plano fiscal, ambas figuras societarias, reciben beneficios fiscales. Las El pueden tributar por el Régimen Simplificado de Tributación hasta por 10 años sin tope de ingresos (MEXICO, SECOFI, 1995); en el caso de las AIE, están exentas del pago del Impuesto de Transmisiones Patrimoniales y de los Actos Jurídicos Documentados; tampoco tributarán por el Impuesto sobre Sociedades, por la parte de base imponible imputable a los socios residentes en territorio español (España. Ministerio de Hacienda, 2004). Tanto las E.I. como las A.I.E., imputan a sus socios tanto los beneficios, como las pérdidas en el ejercicio de sus actividades mercantiles.

\section{Conclusión}

La promulgación del decreto presidencial 07/05/1993 (y su reforma del $30 / 05 / 1995$ ) constituye per se un importante avance normativo, para que las mipymes en México conformen alianzas operativas y estructurales para elevar su producción y comercialización, y al mismo tiempo puedan disminuir sus costos operativos, sin que esto suponga menoscabo alguno de su identidad empresarial.

Lamentablemente el texto normativo presenta ciertas lagunas (por ejemplo, no define en ningún artículo la figura de la empresa integradora) y alguna que otra

6 Para mayor información sobre los programas y subvenciones consultar: http://www.bidasoaactiva.com/descargas/ventanilla/aie-3.doc 
inconsistencia (en el primer decreto establece que sólo micros, pequeñas y medianas empresas conformen una E.I.; en el decreto reformador, permite la participación de cualesquiera otra empresa o institución pública, hasta por el 25\% del capital social).

Ante estas situaciones teóricas, que en la práctica generan no pocas confusiones prácticas, es saludable acudir a figuras asociativas similares en países que cuentan con una amplia experiencia en el tema. Es así, como a través del derecho comparado (al igual que hacen nuestros legisladores), que podemos conocer de otras realidades similares a la nuestra, para posteriormente (si se presenta el caso), adaptarlas a las necesidades nacionales, y por ende establecer ciertos paralelismos jurídicos que nos ayuden a entender y mejorar la producción propia.

En este contexto, las semejanzas de la Empresa Integradora con el Agrupamiento de Interés Económico es más evidente, que aquélla con el Consorcio Italiano.

El común denominador de la actividad mercantil (y por ende empresarial), identifica tanto a las E.I. como a las A.I.E., cuyos objetivos son facilitar la creación y desarrollo de proyectos económicos de colaboración inter-empresarial mediante una estructura horizontal, a través de una figura societaria mercantil, que permita a grupos productivos y comerciales, especialmente pymes, competir frente a grandes empresas o grupos empresariales internacionales. Por el contrario, el consorcio mantiene una ambivalente función, esto es preponderantemente social, y en menor medida, empresarial.
La defensa a ultranza de la identidad empresarial de los socios (tanto de los socios/agrupados como de los socios/integrados), frente a la agrupación o integración empresarial, es otra semejanza en la normativa de las E.I. y las A.I.E.; esta situación es altamente contrastante con la figura italiana, donde el consorcio es el representante que identifica frente a terceros a la mipyme representada.

Ambos elementos, el carácter mercantil y la identidad de los socios por encima de la empresa agrupadora/integradora, advierten la influencia que ejerce la A.I.E. sobre la E.I. Pero es en el ámbito fiscal cuando observamos con mayor claridad que ambas figuras societarias, no permiten (y mucho menos facilitan) la supra-administración contable por parte de la empresa agrupadora/integradora que sustituya a todas y cada una de sus socias.

La intención del legislador europeo y mexicano, parece ser la protección de las mipymes agrupadas/integradas, para que éstas en cualquier momento conozcan y salvaguarden su realidad operativa, productiva, administrativa y fiscal, frente a la empresa agrupadora/integradora.

Si esto no fuera así, el alto grado de preparación técnica del personal integrante de las empresas agrupadoras/integradoras aunado al pormenorizado conocimiento de todas y cada una de las empresas agrupadas/integradas, podría llegar a ser utilizado en contra de estas últimas a favor de la primera, con el consiguiente menoscabo económico y social para los asociados.

Nuevamente aquí se observa el paralelismo que guardan los textos normativos de la AIE con la El, y que vuelve a ratificarse cuando se califica en varios de sus 
Empresas integradoras de México: Influencias europeas

Rodríguez Monroy, Carlos y Fernández Chalé, Lizbeth Noemí

párrafos, el carácter auxiliar las figuras de la empresa integradora y el agrupamiento de interés económico. Por el contrario, el consorcio italiano, concede mayores prerrogativas a favor del consejo consorcial, tanto en administración como en control y representación en detrimento de los socios-consorciados.

Por todos estos motivos, consideramos que el consorcio no parece ser la figura que influyó al legislador mexicano a la hora de crear la figura asociativa de la empresa integradora; quizás lo que sí inspiró fue lograr los resultados que se dieron en las PYMES italianas, y puestos a buscar la fuente que dio origen a esta figura, sería más plausible hacerlo con el agrupamiento de interés económico, cuyo perfil jurídico parece tener más semejanzas que diferencias con la mexicana empresa integradora.

\section{Referencias Bibliográficas}

Jefatura del Estado (1991). "Ley 12/1991, de 29 de abril, de Agrupaciones de Interés Económico". Boletín Oficial del Estado, $n^{\circ} 103$ del 30/04/1991. España.

Ministerio de Hacienda (2004). "Texto Refundido de la Ley de Impuesto sobre Sociedades" Real Decreto Legislativo 4/2004 de fecha 5 de marzo. Boletín Oficial del Estado, $\mathrm{n}^{\circ} 61 \mathrm{del}$ 11/03/2004. España.

Gómez, Calero Juan (1993). Las agrupaciones de interés económico. J.M. Bosch Editor. Barcelona, 281p.

Hualde, Alfredo (2002). "El territorio como configuración compleja en las relaciones entre educación y trabajo". En: Ibarrola, M. (Coord). Desarrollo local y formación: hacia una mirada integral de la formación de los jóvenes para el trabajo. Organización Internacional del Trabajo (OIT), CINTERFOR. 39-66.

II Codice Civile Italiano (1942). Disposizioni Di Attuazione e Transitorie. Regio Decreto 30 marzo 1942, n. 318 - Disposizioni per 1'attuazione del Codice Civile e disposizioni transitorie. Libro Quinto del Lavoro. Titolo X. Della Disciplina Della Concorrenza e dei Consorzi. Edizione 1999-2000 in corso di aggiornamento. Italia.

Secretaría de Comercio y Fomento Industrial, SECOFI (1988). Ley Federal para el Fomento de la Microindustria. Diario Oficial de la Federación 26/01/ 1988. Tomo CDXII, No. 17. México, p.10-16.

Secretaría de Comercio y Fomento Industrial, SECOFI (1993a). "Decreto que promueve la organización de Empresas Integradoras" Diario Oficial de la Federación 07/05/1993. Tomo CDLXXVI, No. 4. México, p.37-39.

Secretaría de Comercio y Fomento Industrial, SECOFI (1993b). "Ley de Inversión Extranjera" Diario Oficial de la Federación 27/12/1993. Tomo CDLXXXIII, No. 19. México, p.92-99.

Secretaría de Comercio y Fomento Industrial, SECOFI (1995). "Decreto que modifica al diverso que promueve la organización de empresas integradoras" Diario Oficial de la Federación 07/05/1995. Tomo D, No. 20. México, p. 9-10.

Secretaría de Comercio y Fomento Industrial, SECOFI (1996). "Decreto por el que reforman, adicionan y derogan la Ley de Inversión Extranjera", Diario Oficial de la Federación, 24/12/ 1996, Tomo DXLIV, No. 12. México.

Monge G., Ángel Luis (1993). Las agrupaciones de Interés Económico. Madrid: Tecnos D.L. 
Orozco Feliger Carlos (1999). "La configuración de empresas integradoras". Resumen de Conferencia. Revista digital. Marketing Global. Instituto Tecnológico de Estudios Superiores de Occidente. Año III, Epoca1, Número 12, Julio. [en linea] www.mktglobal.iteso.mx/numanteriores/1999/julio99/Julio995.html - 19k - [consultado enero 2007]

Perera Sabido, David (2000). Aspectos Jurídicos, Productivos, Fiscales y Estratégicos de las Empresas Integradoras. Ediciones Fiscales ISEF, $1^{\text {a }}$ Ed. México, 143p.
Rueda Peiró, Isabel (1997). Las Empresas Integradoras en México. Ed. Siglo XXI. México

Secretaría de Hacienda y Crédito Público (1999). "Decreto por el que se expide la Ley de Protección al Ahorro Bancario, y se reforman, adicionan y derogan diversas disposiciones de las leyes del Banco de México, de Instituciones de Crédito, del Mercado de Valores, y para Regular las Agrupaciones Financieras". Diario Oficial de la Federación 19/01/1999. Tomo DXLIV, No. 12. México, p.7-33. 